\title{
Robust and Automatic Calibration Method for 3D Freehand Ultrasound
}

\author{
François Rousseau, Pierre Hellier, and Christian Barillot \\ IRISA, Université Rennes 1, INRIA, CNRS, \\ Campus de Beaulieu, Rennes, France \\ Francois.Rousseau@irisa.fr, \\ http://www.irisa.fr/vista
}

\begin{abstract}
This paper describes a new robust and fully automatic method for calibration of three-dimensional (3D) freehand ultrasound. $3 \mathrm{D}$ Freehand ultrasound consists in mounting a position sensor on a standard probe. The echographic B-scans can be localized in 3D, and can be compounded into a volume. However, especially for quantitative use, this process dramatically requires a calibration procedure that determines its accuracy and usefullness. Calibration aims at determining the transformation (translations, rotations, scaling) between the coordinates system of the echographic images and the coordinate system of the localization system. To calibrate, we acquire images of a phantom whose 3D geometrical properties are known. We propose a robust and fully automatic calibration method based on the Hough transform and robust estimators. Experiments have been done with synthetic and real sequences, and this calibration method is shown to be easy to perform, accurate, automatic and fast enough for clinical use.
\end{abstract}

\section{Introduction}

As it is mostly non-invasive and has a real time capability and a relatively low cost nature, 2D ultrasound is popular. Its major drawback is its weak capability of issuing quantitative accurate morphometric information [5]. In fact, conventional ultrasound exams are limited by 2D viewing, and follow-up studies are then not easily reproducible. 3D ultrasound imaging overcomes these limitations. In addition, it facilitates extensive investigation and allows accurate measurements of organ volumes.

$3 \mathrm{D}$ freehand ultrasound imaging is a way of acquisition in which a localization system is fixed to a $2 \mathrm{D}$ probe. The localization system, which can be magnetic, optic, acoustic or mechanical, continuously gives the position and orientation of the probe. Using the known positions and orientations of each $2 \mathrm{D}$ scan, a $3 \mathrm{D}$ image can be reconstructed. Freehand systems suffer from possible motion during the exam (organ, respiration, heart beat etc.), a lesser accuracy with respect to 3D US using a 3D probe, and a need of a geometrical calibration. However, a freehand system is cheap and close to clinical routine so it can readily be applied in many interventions and surgeries. Contrary to mechanical probes, it also allows the examination of large organs. 
To avoid geometrical artefacts and distortions in 3D freehand ultrasound, orientations and locations of the acquired 2D scans have to be accurately known which requires accurate calibration. This is of particular importance if the 3D US data are to be used in image guided interventions, when the geometrical precision becomes crucial.

Calibration is needed to correctly localize an image in $3 \mathrm{D}$ space and embraces a temporal and spatial calibration. The aim of temporal calibration is to match the position information with the echographic frames. Spatial calibration consists of determining the transformation between pixels in the US image and points in $3 \mathrm{D}$ space. Whatever the localization system, calibration is crucial because it has a significant impact on the quality of reconstruction. The calibration procedure has to be as easy as possible to perform in clinical context, i.e., automatic, user friendly, easy to operate, robust and fast.

We address in this paper two issues related to 3D freehand ultrasound : design of an automatic, robust, fast and reliable spatial calibration procedure; experiments with synthetic and real sequences and a 3D ultrasound calibration phantom. The paper is organized as follows. Section 2 presents the spatial calibration problem and a brief state of art. The proposed calibration method is described in Section 3 . Section 4 presents the experiments and the results.

\section{What Is Spatial Calibration?}

\subsection{Formulation}

The spatial calibration problem can be formulated as [9]:

$$
\mathbf{x}_{c}=T_{c} T_{t} T_{r} \mathbf{x}_{r}, \text { with } \mathbf{x}_{r}=\left(s_{x} u, s_{y} v, 0,1,\right)^{T}
$$

where $T_{r}$ denotes the rigid transformation from $\mathrm{B}$ scan to receiver coordinates, $T_{t}$ the rigid transformation from receiver to transmitter coordinates, $T_{c}$ the rigid transformation from transmitter to phantom coordinates, $u$ and $v$ are the image pixel coordinates, $s_{x}$ and $s_{y}$ scaling factors (see Figure 1).

Performing the calibration amounts to estimating the matrix $T_{r}$ (3 translations and 3 rotations) and the scaling coefficients $s_{x}$ and $s_{y}$. The calibration is generally carried out in the following way: after scanning a phantom whose $3 \mathrm{D}$ geometrical properties are known, the calibration is based on this geometry being recovered in the sequence of ultrasound images.

\subsection{Related Work}

Spatial calibration methods can be classified in three groups according to the phantom used: wire phantom, multimodality registration phantom, single-wall phantom.

The most popular point based calibration method is the cross-wire method proposed by Detmer et al. [4] : the intersection of two wires is scanned. Calibration parameters are estimated by minimizing the difference between the 


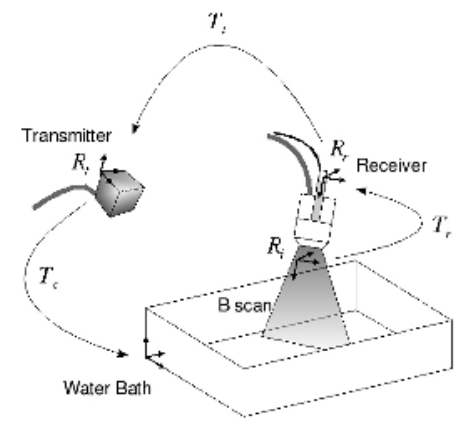

Fig. 1. Coordinate systems : $R_{i}$ (image), $R_{r}$ (receiver), $R_{t}$ (transmitter), $R_{c}$ (reconstruction volume, here the water bath).

recorded positions of the intersection point $\mathbf{P}=\left(P_{x}, P_{y}, P_{z}\right)$ and its mean position $\overline{\mathbf{P}}=\left(\overline{P_{x}}, \overline{P_{y}}, \overline{P_{z}}\right)$. Using wire phantom, there are also fiducial based methods proposed by [1], 2], 8]. Calibration parameters are estimated by detecting points of interest of a specific phantom in ultrasound images and by minimizing a least square equation.

Blackall et al. proposed [1] an image registration approach to calibrate freehand $3 \mathrm{D}$ ultrasound. The idea is that a consistent calibration gives an optimal similarity measure between the ultrasonic images of the phantom and a 3D model of this one (CT or MR volume).

Finally, Prager et al. [9] use the wall of a water bath to perform the spatial calibration. The water bath plane appears to be a straight line in a B-scan. An extracted line defines two points on the phantom's plane. Each pixel on the line should satisfy: $(x, y, 0,1)^{T}=T_{c} T_{t} T_{r}\left(s_{x} u, s_{y} v, 0,1\right)^{T}$. The zero component of this equation gives one equation to determine the calibration parameters. Given a line defined by two pixels, each B-scan gives two equations.

\subsection{Motivations of Our Work}

Table 1 summarizes advantages and drawbacks of existent calibration methods. We take into account differents criteria to characterize calibration method: a) the cost of the needed material to perform calibration (phantom, optical tracker, etc.), b) the simplicity of the used phantom, the easiness to built it, c) the ease to perform the calibration, d) the quickness of the calibration process, e) the needed of external intervention.

So, there is a clear need for a cheap, robust, fast and fully automatic procedure. In order to meet the requirement of 3D ultrasound on routine applications, we propose a calibration technique which gather all this properties. This is particularly needed when the attachement between the probe and the localization system cannot be stable over time. 
Table 1. Advantages and drawbacks of existent calibration methods.

\begin{tabular}{|l|c|c|c|c|c|}
\hline Calibration Method & Cost & Ease to built & Ease to use & Quickness & Automatic \\
\hline Cross-wire & + & + & - & - & no \\
Fiducial based & + & - & + & - & no \\
Single-wall phantom & + & + & + & - & no \\
Image registration approach & - & + & - & - & no \\
\hline
\end{tabular}

\section{The Proposed Calibration Method}

\subsection{Phantom}

With the aim of carrying out a simple, fast and completely automatic calibration, we have chosen to use a plane phantom. This type of phantom is easy to built and to use. Moreover, it provides in each image a strong, straight line which can be automatically detected with accuracy. This kind of phantom does not need any registration with optical tracker in the reconstruction coordinate system.

\subsection{Extraction}

A point set in each image coordinate system has to be extracted, and this point set should correspond to a plane. The highest gradient and highest luminance points are retained by adaptive thresholding. We combine these two features because either intensity or gradient information would not be sufficient for a reliable extraction when the probe moves too fast during the acquisition (see figure 2(b)). To reject outliers in each image (see figure 2(b)), the Hough transform is used [6]. The Hough transform permits to isolate features of a particular shape within an image. In each B-scan, a line is extracted. As the Hough transform is robust, each extracted line is used to reject outliers which may contains the point set.

\subsection{Formulation}

With the aim of doing a calibration method easily usable in clinical routine, no localization system is used to determine the position of the plane in the reconstruction volume. As a consequence, the parameters of the plane have to be estimated. Therefore, equation (11) becomes: $\mathbf{x}_{t}=T_{t} T_{r} \mathbf{x}_{r}$.

Calibration parameters are estimated to minimize the Euclidian distance between the plane and the points of interest. We propose here two differents ways to do so: a distance criterion expressed in 3D coordinate system:

$$
\hat{T}_{r}=\arg \min _{T}\left\{\frac{1}{2} \sum_{i=1}^{N} d\left(\pi, M_{i}\right)^{2}\right\}
$$

where $\mathrm{N}$ is the cardinal of the point set, $d(\pi, M)$ is the Euclidian distance between the plane $\pi$ and the point of interest $M_{i}$ in 3D space; or a 2D distance expressed in each B-scan: 


$$
\hat{T}_{r}=\arg \min _{T}\left\{\frac{1}{2} \sum_{i=1}^{N} d\left(P(\pi), m_{i}\right)^{2}\right\}
$$

where $d(P(\pi), m)$ is the Euclidian distance between the plane $\pi$ projected in the image and the point of interest $m_{i}$ in US images. This complicated non-linear cost function has to be optimized over a nonlinear parameter space.

The first formulation using 3D euclidian distance is quite intuitive because the point set has to be aligned with the wall of the water bath (a 3D object). In visual reconstruction domain where $3 \mathrm{D}$ structure and viewing parameters (camera pose or/and calibration) have to be jointly estimated, the cost function is defined in images using a basic image projection model [12]. The reason is that the $3 \mathrm{D}$ coordinate frame is itself uncertain, as it can only be located relative to uncertain reconstructed features or cameras. The two formulations will be compared in the experiment section.

\subsection{Robustness}

To be automatic and robust, the method includes outlier modeling both in the extraction and optimization.

The least square minimization method might be sensitive to outliers due to speckle noise in US image. Using Hough transform during the extraction step, we explicitely use temporal coherence between each B-scan. Continuity of probe motion should lead to a smooth variation of line parameters. To reject image with incorrect line detection, a likelyhood threshold test based on line parameters is performed between two successive B-scans.

To improve robustness in the optimization step, an outlier rejection algorithm is used. It is not possible to use a classical 3D outliers rejection algorithm because calibration parameters estimation need a complete set of the probe motions [9]. A robust estimator has to be used on each image to be as accurate as possible, using 3D information. We use Least Trimmed Squares estimator (LTS) 10]. The LTS estimator $\mathcal{T}$ minimizes a criterion $\mathcal{C}$ with $r_{1}^{2} \leq r_{2}^{2} \leq \cdots \leq r_{N}^{2}$ the ordonated remainders, and $h$ the number of points used for the estimation: $\hat{T}=\arg \min _{T} \sum_{i=1}^{h} r_{i}^{2}$, with $\frac{N}{2} \leq h \leq N$. For each point, computed remainder is equal to the distance between the plane and the point. $3 \mathrm{D}$ information is introduced using all the sequence in the 2D rejection algorithm. LTS estimator is used because there is only one parameter to tune (the percentage of outliers in each B-scan) contrary to the M-estimators where rejection parameters have to be carefully tuned.

\subsection{Optimization}

Plane parameters and calibration parameters are dependent each others. The optimization algorithm has to take into account this dependency. Using LevenbergMarquardt algorithm [7], the minimization process consists in estimating all the calibration parameters and coefficients plane together. 


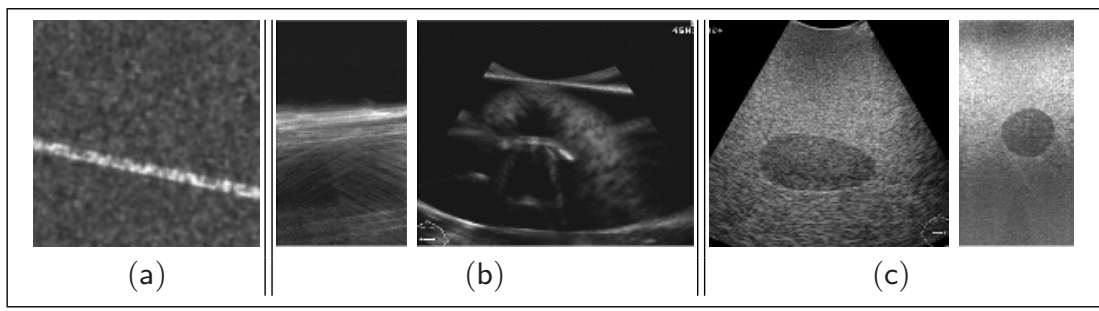

Fig. 2. Representative images of US Sequences. In (a), added speckle noise synthetic image; in (b), real images of the water bath; in (c), phantom B-scans.

To shorten the computation time, we propose a hierarchical algorithm. The complexity of the algorithm only depends on the number of points of interest used to minimize $f$. Similarly to multiresolution algorithm ([3]), we divide the optimization procedure into several stages: $N$, the number interest points, is increased at each hierarchical iteration.

\section{Calibration Experiments}

Our algorithm has been tested with synthetic and real sequences. Synthetic images are interesting because the calibration parameters to be recovered are exactly known. Thus, we can evaluate the precision and the repeatability of the calibration algorithm. Moreover, it is possible to test the intrinsic precision of our method, because many sources of errors are eliminated (localization errors of each B scan, errors due to beam thickness, etc.).

\subsection{Synthetic Sequences}

Description. The method was tested with three synthetic sequences with different calibration parameters sets. Each sequence contains 121 images (256 by 256 pixels): one reference image and 20 images per motion (three translations and three rotations). Ultrasound images are classically corrupted by the presence of multiplicative speckle noise. To test the algorithm with more realistic sequences, we add speckle noise to images. Fully developped speckle can be modeled with the Rayleigh law. The Rayleigh probability density function is defined by the following $f(x)=\frac{x}{\sigma^{2}} e^{-\frac{x^{2}}{2 \sigma^{2}}}, x \geq 0$. Figure 2(a) shows a B-scan extracted from synthetic noisy sequence.

Results. For each sequence, the algorithm has been tested with 20 different initialization domains: $\pm 40(\mathrm{~mm})$ for translation, $\pm 40^{\circ}$ for rotations and $\pm 50(\%)$ for scale factors. Concerning the hierarchical aspect, experiments have showed that it is optimal to divide the optimization stage by three. We cannot describe this intensive experiment here for sake of brievity. Calibration results are summarised in table 2. 
Table 2. Calibration results : Mean error and standard deviation are given for translations, rotations and scaling factors. The results for translations are in $\mathrm{mm}$, for rotations in degrees, for scaling factors in $\mathrm{mm} /$ pixel.

\begin{tabular}{||l|c|c|c||}
\hline Calibration Method & 2D criterion & 3D criterion & Stradx \\
\hline Mean Error and Std. Dev.(mm) & $0.9594(0.1137)$ & $0.5454(0.0952)$ & $1.4874(0.7293)$ \\
\hline Mean Error and Std. Dev.(deg) & $0.0068(0.0010)$ & $0.0015(0.0006)$ & $0.0060(0.0037)$ \\
\hline Mean Error and Std. Dev.(mm/pix) & $0.0014(0.0004)$ & $0.0021(0.0003)$ & $0.0080(0.0091)$ \\
\hline
\end{tabular}

We have tested the three synthetic noisy sequences with the Stradx software 9]. StradX may be considered as a reference in the 3D freehand ultrasound domain. The results are summarised in table 2 With the presence of fullydevelopped speckle in each sequence, line detection parameters in Stradx have to be tuned, otherwise Stradx algorithm could fail to converge. Thus, StradX has been tested with different parameters sets. We tuned StradX parameters to the best of our expertize. These results indicate that even with noisy sequences and a large initialization domain, the calibration appears to be accurate. Considering the three synthetic sequences, one can say that the two proposed methods seems slightly more successful than StradX. Even if synthetic sequences allow well to evaluate a calibration method because the ground truth is exactly known, tests on real data are needed.

\subsection{Real Sequences}

Acquisition. Real sequences have been acquired with ATL ultrasound scanner (HDI 5000) and a magnetic localization system (Pc-Bird, Ascension Technology) attached to the probe. This type of device provides a flexible and inexpensive solution, and has already been successfully widely used for 3D ultrasound (e. g. [4], [9]). The probe used in this study was a hand-held linear scanhead (ATL, L 12-5). The size of the B-scan is 228 by 485 (pixels).

Calibration Evaluation. To evaluate the reconstruction accuracy, a 3D ultrasound calibration phantom (CIRS, http://www.cirsinc.com) was scanned. A test object with known volume $\left(6.6162 \mathrm{~mm}^{3}\right)$, was reconstructed. Figure (2) (c) shows B-scans of the phantom. Therefore, we can compare the volume given by the manufacturer with the estimated volume for different calibration methods. To evaluate the calibration reproductibility, we use a criterion proposed by [9] : $\Delta \mathbf{x}^{t}=\left\|T_{r 1} \mathbf{x}_{r}-T_{r 2} \mathbf{x}_{r}\right\|$, where $\mathbf{x}_{r}$ is a corner point of B-scan (this expression includes the scaling factors), $T_{r 1}$ and $T_{r 2}$ two calibration transformations for the same ultrasound sequence. A small $\Delta \mathrm{x}^{t}$ indicates a good reproductibility.

Results. Five sequences have been used and some of these have a quite poor quality (see figure 2(b)). These sequences show clearly the need of a robust and fully automatic calibration procedure. Computation time is under 5 minutes. Table 3 summarizes reproductibility results using the criterion $\Delta \mathrm{x}^{t}$ and accuracy 
Table 3. Volume measurements and reproductibility measure. The ratio between the mean estimated volume and the true volume is given into brackets.

\begin{tabular}{||l|c|c|c||}
\hline Volume & 2D criterion & 3D criterion & Stradx \\
\hline Mean volume and Std. Dev. $(\mathrm{mm} 3)$ & $6.0531(0.3401)$ & $5.9757(0.1892)$ & $5.7257(0.5708)$ \\
\hline \hline Reproductibility $\left(\Delta \mathbf{x}^{t}\right)$ & 2D criterion & 3D criterion & Stradx \\
\hline Mean Error and Std. Dev. $(\mathrm{mm})$ & $0.9112(0.3810)$ & $0.8928(0.3439)$ & $0.9594(0.4351)$ \\
\hline
\end{tabular}

evaluation with volume measurement method. The results indicate that the two proposed formulations are quite equivalent and perform better than the Stradx method in terms of reproductibility and precision.

\section{Conclusion}

We present in this paper a novel robust, fast and fully automatic method to calibrate a 3D freehand ultrasound system. The calibration parameters are determined by aligning iteratively a plane phantom and a set of points of interest extrated in 2D images. The points extraction step uses the Hough Transform. Robustness is obtained by verifying 3D spatial coherence of the Hough Transform coefficients and using an outlier rejection process for each B-scan. The proposed method is fully automatic which a key point to use 3D freehand ultrasound in clinical applications. We investigate a 2D and 3D formulation of the calibration problem. Reproductibility and accuracy have been evaluated with synthetic and real sequences. The different formulations have been compared with StradX software which can be considered as a reference in this domain. The performance of the proposed method was significantly better than StradX. 3D approach is slightly more accurate than 2D approach, yet more time consuming. Our method has been shown to be robust, accurate and fast enough for clinical use.

Acknowledgment. We would like to thank François Chaumette from IRISAINRIA for fruitful discussions.

\section{References}

1. Blackall, J. M. and Rueckert, D. and Maurer Jr, C. R. and Penney, G. P. and Hill, D. L. G. and Hawkes, D. J. : An image registration approach to automated calibration for freehand 3D ultrasound. MICCAI, 462-471 (2000).

2. Bouchet, L.G. and Meeks, S.L. and Goodchild, G. and Bova, F.J. and Buatti, J.M. and Friedman, W.A.: Calibration of three-dimensional ultrasound images for image-guided radiation therapy. Physics in Medicine and Biology, 46(2), 559-577 (2001).

3. Burt, P.J.: The Pyramid As A Structure For Efficient Computation. Multiresolution image processing and analysis, 12, 6-35 (1984) 
4. Detmer, P. R. and Bashein, G. and Hodges, T. and Beach, K. W. and Filer, E. P. and Burns, D. H. and Strandness Jr, D. E. : 3D ultrasonic image feature localization based on magnetic scanhead tracking : in vitro calibration and validation. UMB, 20(9), 923-936 (1994).

5. Fenster, A. and Downey, D. B. and Cardinal, H. N. : Three-dimensional ultrasound imaging. Physics in medicine and biology, 46, 67-99 (2001).

6. Hough, P.V.C.: Machine Analysis of Bubble Chamber Pictures. International Conference on High Energy Accelerators and Instrumentation, CERN (1959).

7. Moré, J. J.: The Levenberg-Marquardt algorithm: implementation and theory. Proceedings of the Biennial Conference on numerical analysis, 105-116 (1978).

8. Pagoulatos, N. and Haynor, D. R. and Kim, Y.: A fast calibration method for 3-D tracking of US images using a spatial localizer. UMB, 27(9) 1219-1229 (2001).

9. Prager, R. W. and Rohling R. N. and Gee A. H. and Berman L. : Rapid calibration for 3-D freehand ultrasound. UMB, 24(6), 855-869 (1998).

10. Rousseeuw, P. J. and Leroy, A. M. : Robust regression and outlier detection. Wiley Series in Probability and Mathematical Statistics (1987).

11. Sato, Y. and Nakamoto, M. and Tamaki, Y. and Sasama, T. and Sakita, I. and Nakajima, Y. and Monden, M. and Tamura, S.: Image guidance of breast cancer surgery using 3-D ultrasound images and augmented reality visualization. TMI 17(5), 681-693 (1998).

12. Triggs, B. and McLauchlan, P. and Hartley, R. and Fitzgibbon, A.:Bundle Ajustment, A modern synthesis. Vision Algorithms: Theory and Practice (2000). 